\title{
ARTICLE OPEN \\ Patients' perspectives on the impact of a new COPD diagnosis in the face of multimorbidity: a qualitative study
}

\author{
Sameera Ansari ${ }^{1}$, Hassan Hosseinzadeh ${ }^{1}$, Sarah Dennis ${ }^{1,2}$ and Nicholas Zwar ${ }^{1}$
}

BACKGROUND: Chronic obstructive pulmonary disease (COPD), a major cause of morbidity and mortality worldwide, often occurs in the presence of comorbidities, which may influence experience and management of the disease. No prior research seems to have gained perspectives of newly diagnosed primary care COPD patients in the context of multimorbidity.

AIMS: This qualitative study aimed to explore the impact of a new diagnosis of COPD in the context of multimorbidity and also sought to gain a better understanding of how patients react to the diagnosis and incorporate it into their lives.

METHODS: Participants were identified from a cohort of primary care patients with multimorbidity recently diagnosed with COPD. Data was collected via semi-structured interviews from nine male and eight female participants. Thematic analysis was performed and the data interpreted from a constructivist perspective.

RESULTS: Five core themes regarding COPD were induced: (i) reaction to diagnosis, (ii) impact on function and health behaviour, (iii) factors influencing self-management capacity, (iv) healthcare utilisation and (v) interplay of comorbidities. Most participants had difficulty recognising the importance of COPD and its long-term implications. For many, the salience of another chronic condition outweighed COPD. Self-management capacity and utilisation of healthcare services were challenged by low prioritisation of COPD among other comorbidities.

CONCLUSIONS: This study provides an insight into how primary care patients feel about being diagnosed with COPD, as well as their prioritisation of the disease in the context of multimorbidity. It highlights the need for tailored education and personalised management incorporating patients' perspectives in primary care.

npj Primary Care Respiratory Medicine (2014) 24, 14036; doi:10.1038/npjpcrm.2014.36; published online 14 August 2014

\section{INTRODUCTION}

Chronic obstructive pulmonary disease (COPD) is currently the fourth leading cause of mortality worldwide and is expected to become the third by the next decade. ${ }^{1}$ In Australia, $4 \%$ of all deaths in 2006 were attributed to COPD. ${ }^{2}$ Primarily caused by exposure to noxious particles or gases, COPD is an independent risk factor for lung cancer ${ }^{3}$ and causes a progressive, irreversible deterioration in patients' quality of life. ${ }^{4}$

COPD often occurs in the presence of other chronic health conditions, known as comorbidities, ${ }^{5}$ which might be related (concordant), such as asthma or depression, or unrelated (discordant), like diabetes or fibromyalgia. ${ }^{6}$ Irrespective of being concordant or discordant, comorbidities may influence experience and management of the disease by patients. ${ }^{7}$ This coexistence of two or more chronic health conditions in the same individual is known as multimorbidity, ${ }^{8}$ which is the rule rather than the exception in primary care. ${ }^{9}$ Multimorbidity contributes to higher healthcare utilisation with associated increase in time and costs, especially in older adults. ${ }^{10}$ In 2008, multimorbidity data extraction from two Australian primary care practices revealed respiratory diseases to be the third most common domain. ${ }^{11}$

There are numerous clinical outcome measures available to quantify the health-related impact of COPD and its comorbidities, ${ }^{12,13}$ but a need exists for qualitative research to better understand coping strategies in patients with COPD. ${ }^{14}$ Though there is a growing amount of literature about the impact of comorbidities on COPD and vice versa, ${ }^{7,15-18}$ research focused on patients' experience of these interactions is limited. ${ }^{19,20}$ While some studies have explored patients' attitudes and beliefs of living with the disease, ${ }^{21-23}$ there is a dearth of qualitative research regarding patients' perspectives on the impact of COPD diagnosis. ${ }^{24}$ In particular, no studies seem to have focused on the experience of newly diagnosed COPD patients with comorbidities, especially in primary care.

Our qualitative research study is an attempt to fill this gap by exploring primary care patients' perspectives regarding the impact of a new diagnosis of COPD in the context of multimorbidity.

\section{MATERIALS AND METHODS}

\section{Sampling and recruitment of participants}

Participants for this qualitative study were drawn from a cohort of 254 patients who had taken part in the PELICAN (Primary care EarLy Intervention for Copd mANagement) project, $^{25}$ a recent randomised controlled trial of case finding. The PELICAN participants were aged between 40 and 85 years, had a history of smoking and were recruited from primary care practices across Sydney. As shown in Figure 1, 140 of the 254 participants had been diagnosed with COPD during the trial, which is considered a new diagnosis for the purpose of the qualitative study. Among the 140, 117 participants met the inclusion criteria of this study by having one or more preexisting comorbidity along with a new COPD diagnosis (in the past 24 months).

Using maximum variation type of purposive sampling to ensure diversity, the eligible sample of 117 was narrowed down to 30 prospective participants based on gender, age, comorbidity count, severity of COPD

\footnotetext{
${ }^{1}$ School of Public Health and Community Medicine, UNSW Medicine, UNSW Australia, Sydney, NSW, Australia and ${ }^{2}$ Clinical and Rehabilitation Sciences, Faculty of Health Sciences, The University of Sydney, Sydney, NSW, Australia.

Correspondence: S Ansari (sameera.ansari@unsw.edu.au)

Received 30 January 2014; revised 21 June 2014; accepted 1 July 2014
} 
(as defined by GOLD (Global initiative for Chronic Obstructive Lung Disease $)^{4}$ ), level of education, employment, social support and smoking status. Of the 30 potential participants for the study, 4 were not reachable. Among the remaining 26 approached, 4 were reported to be deceased, 3 declined and 19 consented to participate. There was one participant who dropped out at the beginning of his interview citing personal reasons. Another participant had to be excluded as it was revealed during the interview that he had been diagnosed with COPD before the PELICAN trial.
The final study sample comprised nine male and eight female participants aged between 43 and 84 years. Their demographic characteristics are outlined in Table 1.

Data collection and analysis

Verbal and written consent was obtained from the participants and they were informed of their right to withdraw from the study at any time. Due

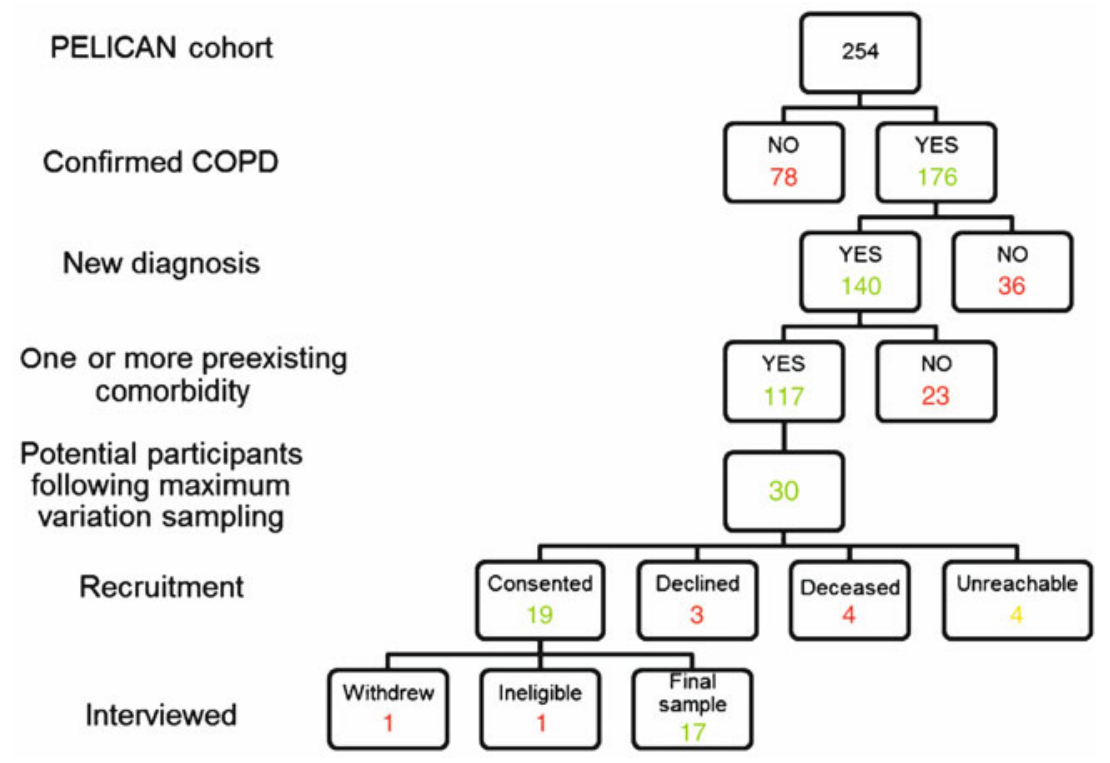

Figure 1. Recruitment of participants.

Table 1. Demographic characteristics of participants $(N=17)$

Code Participant characteristics

F1 Female, 48 years, lives alone. Completed some high school, unemployed. COPD GOLD I, 14 months since diagnosis, 3 comorbidities, current smoker.

F2 Female, 62 years, lives alone. Completed some high school, volunteer. COPD GOLD II, 8 months since diagnosis, 3 comorbidities, ex-smoker.

F3 Female, 43 years, lives with spouse and children. Tertiary education, student. COPD GOLD I, 19 months since diagnosis, 5 comorbidities, ex-smoker.

F4 Female, 80 years, lives with spouse. Completed some high school, retired. COPD GOLD II, 15 months since diagnosis, 7 comorbidities, ex-smoker.

M5 Male, 77 years, lives with spouse and child. Vocational training, retired. COPD GOLD I, 20 months since diagnosis, 5 comorbidities, ex-smoker.

M6 Male, 84 years, lives with grandchild. Tertiary education, retired. COPD GOLD II, 19 months since diagnosis, 4 comorbidities, ex-smoker.

F7 Female, 66 years, lives with spouse. Tertiary education, retired. COPD GOLD II, 20 months since diagnosis, 5 comorbidities, quit smoking following COPD diagnosis.

F8 Female, 59 years, lives alone. Completed some high school, unemployed. COPD GOLD I, 19 months since diagnosis, 6 comorbidities, quit smoking following COPD diagnosis.

F9 Female, 52 years, lives alone. Completed high school, employed full-time. COPD GOLD I, 24 months since diagnosis, 2 comorbidities, quit smoking following COPD diagnosis and resumed after a year.

M10 Male, 69 years, lives with spouse. Completed some high school, employed full-time. COPD GOLD I, 7 months since diagnosis, 1 comorbidity, current smoker.

M11 Male, 82 years, lives alone. Completed some high school, retired. COPD GOLD I, 14 months since diagnosis, 4 comorbidities, ex-smoker.

M12 Male, 82 years, lives with spouse. Vocational training, retired. COPD GOLD II, 5 months since diagnosis, 5 comorbidities, ex-smoker.

F13 Female, 69 years, lives with spouse. Completed primary school, retired. COPD GOLD I, 20 months since diagnosis, 4 comorbidities, ex-smoker.

M14 Male, 62 years, lives with spouse and children. Completed some high school, self-employed. COPD GOLD I, 24 months since diagnosis, 1 comorbidity, current smoker.

M15 Male, 64 years, lives with spouse. Tertiary education, employed part-time. COPD GOLD I, 16 months since diagnosis, 7 comorbidities, ex-smoker.

M16 Male, 78 years, lives with spouse. Tertiary education, retired. COPD GOLD I, 21 months since diagnosis, 4 comorbidities, ex-smoker.

M17 Male, 56 years, lives with spouse. Tertiary education, employed full-time. COPD GOLD I, 13 months since diagnosis, 1 comorbidity, current smoker.

Abbreviations: COPD, Chronic obstructive pulmonary disease; GOLD, Global initiative for Chronic Obstructive Lung Disease. 
Box 1 Interview guide

1. Could we start by asking about your experience of being diagnosed with a lung condition?

- How did you feel about it then and how do you feel about it now?

- Has this condition changed the way you think about your health?

If yes, in what ways?

2. What do you call the lung condition?

3. How does the lung condition affect your daily activities and/or social participation? (Example)

4. What treatment, if any, are you undergoing for the lung condition?

- How do you financially manage the treatment of the condition?

5. Have you conducted other health professionals besides your GP for the lung condition?

- If yes, who are they?

6. What changes, if any, have you made in your daily life related to the lung condition?

Please give an example of a change.

- What changes have you made in your health-related behaviour?

What has helped and not helped you in this process?

7. Do you have other long-term health conditions?

- If yes, which ones?

- Which do you consider most important and why?

How do these affect your daily life? (Example)

Which condition has the biggest impact?

8. How do you manage your lung condition compared to your other long-term health conditions?

- How does the lung condition affect your other illnesses and vice versa? (Example)

Which illness do you give highest priority? (Example)

- How does this priority affect what you do? For example, when taking medicines, attending appointments with doctors and other health professionals.

Is it expensive to manage the medication costs for all your health conditions?*

9. How easy or difficult is it for you to follow your health providers' advice for treatment of your health conditions? - What makes it easy or difficult? (Example)

- How do you keep track of the various medications you need to take for your health conditions?*

What can health providers do to help you manage your health conditions better? (Example)

10. Is there anything else you would like to tell us regarding your health conditions?

*Added after the first six interviews.

to widespread location of participants across the Sydney region and based on evidence supporting the equivalence of telephone and face-to-face interviewing, ${ }^{26}$ telephone interviews were determined to be the most convenient mode of data collection. A semi-structured interview guide (Box 1) was developed for the study. Of the final 17 participants, 16 were interviewed via telephone while 1 participant was interviewed at home because he was hard of hearing. Data was deemed to be sufficient following these 17 interviews because saturation ${ }^{27}$ had been attained by then. Data collection was conducted by SA and took place through late April to early July 2013. The interviews simulated natural conversation while ensuring the main topics were explored sufficiently. The average duration of the interviews was $30 \mathrm{~min}$. All interviews were digitally recorded and transcribed verbatim.
The research team held regular meetings to analyse every stage of data collection and analysis. Preliminary thematic analysis ${ }^{28}$ and initial coding commenced alongside data collection by listening to the recorded interviews. As a result, the interview guide was slightly modified after the first six interviews by addition of two probing questions. Following transcription, the data was organised and axial coding performed with the aid of NVivo 10 software. Thorough thematic analysis of the coded interviews was done by repeated exposure to the fragmented data. Using a constructivist ${ }^{29}$ approach of data interpretation, the coded narratives of the participants were iteratively analysed by the research team to unravel deeper meaning until a consensus was reached regarding the main themes that made sense to the researchers. A thematic framework of 16 subthemes were induced and then categorised under five core themes. Each transcript was analysed and interpreted separately by at least one other team member to ensure inter-researcher reliability.

The study had ethics approval from the Human Research Ethics Committee of UNSW Australia (HREC 10015).

\section{RESULTS}

Following data analysis, five core themes encompassing participants' perspectives of the impact of COPD diagnosis were induced, namely (i) reaction to diagnosis, (ii) impact on function and health behaviour, (iii) factors influencing self-management capacity, (iv) healthcare utilisation and (v) interplay of comorbidities.

\section{i) Reaction to diagnosis of COPD}

The participants had mixed reactions to being diagnosed with COPD, from emotional to rational, including denial. 'It (COPD diagnosis) was a big shock because the last cigarette I had was back in 1979' -F13, 'It didn't surprise me because l'd smoked for a long time'-M15.

Female participants in their fifties and sixties seemed more concerned about the COPD diagnosis than their male counterparts. Participants in their seventies and eighties had a more positive attitude. They were more resigned towards accepting an addition to the number of preexisting chronic conditions and also seemed the most passive in terms of incorporating COPD management into their lives. 'Let's face it, I'm 80...a lot of it's just getting older. You can't stop old age...I've got what I've got, so that's it'-F4. The diagnosis increased the participants' awareness of their breathing and enhanced their general perception of health.

\section{ii) Impact of COPD on function and health behaviour}

COPD had a functional impact on 12 of the participants due to breathlessness, affecting domestic chores and outdoor mobility. 'I can't really walk fast anymore, I get puffed'-M16.

Social participation was occasionally restricted for the two youngest participants, both females with mild COPD. 'I can struggle doing anything that takes a lot of effort. I don't like big days out with the family'-F3.

The diagnosis seemed to motivate all the participants to modify their health behaviour in terms of trying to increase or keep up physical activity as well as monitoring diet. 'I'm trying to get a bit more active, walk a bit more, just try and do a little bit of exercise, a bit more than I used to...I'm doing something, I'm not just sitting around'-F1.

Of the seven participants who were smokers at the time of COPD diagnosis, three females quit smoking shortly after the diagnosis whereas the others started working towards smoking cessation. 'I've had a couple of goes and it (quitting smoking) is a difficult thing...I'll get there'-M17.

\section{iii) Factors influencing self-management capacity of COPD}

Only six participants knew what their lung condition was called. Those with other lung conditions at the time of diagnosis, such as 


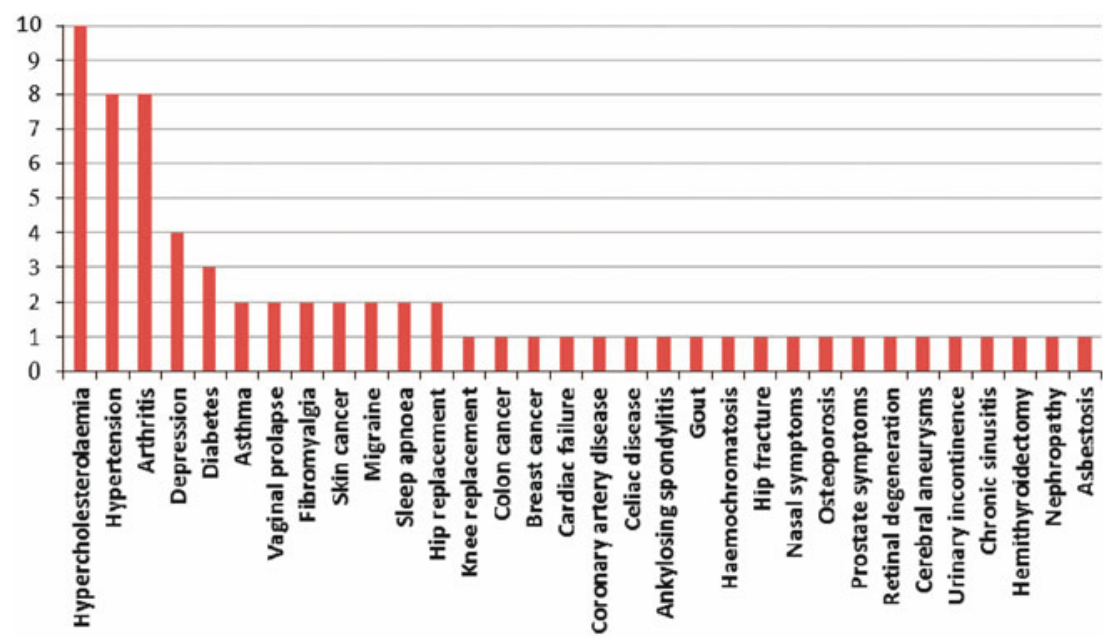

Figure 2. Various comorbidities experienced by the participants. $X$ axis: types of comorbidities, $Y$ axis: number of participants.

asthma, seemed confused about the symptoms and nature of COPD. 'I didn't realise that I had asthma...the two conditions make things very difficult'-M6.

Retired participants had better financial leverage compared with those employed or on other benefits like 'Closing the $\mathrm{Gap}^{\prime 30}$ or 'Newstart'. ${ }^{31}$ It (medicine) would be a lot dearer if I wasn't on the concession sort of deal'-M11. For some, financial constraints impaired comprehensive management of COPD due to low affordability of healthcare. For instance, one participant had to wait more than a year following diagnosis to consult a respiratory physician.

Chronic depression hindered coping with COPD and so did work-related stress, which caused one participant to resume smoking. Some participants felt that their inherent motivational capacity helped in coping with the COPD diagnosis, whereas others relied on family, faith and/or philosophy. 'I always try to look after myself because I know what I've actually got to do as an old bloke like me...I've got kids to look after so I've got to make sure I then look after myself'-M14.

\section{iv) Healthcare utilisation for COPD}

Typically, the participants consulted their family physicians twice a month, mainly to obtain scripts for COPD and other comorbidities. The two participants with asthma visited their healthcare providers more often for consultation regarding COPD. Among the 17 participants, 8 took a daily dose of prescribed inhalers and 3 used an inhaler whenever necessary, whereas 6 of them refrained from using COPD medication in spite of having been advised to do so. 'I didn't have any breathing problems... It (COPD) doesn't affect any activities that I know of'-M5.

With the exception of one participant who switched general practitioners following COPD diagnosis, many others were satisfied with their healthcare providers. Some felt they could better manage their health themselves, whereas one participant voiced the need for personalised plans and support in terms of smoking cessation. 'Maybe a better plan for the smoking...It's individual, instead of lumping everyone in one group 'cause everyone is different' $-F 9$.

Though most participants relied heavily on their healthcare providers for advice and support, there was an apparent lack of communication between the participants and their physicians in terms of COPD. 'I know more about my body than what a doctor does, I know what's the matter with me. And sometimes you can tell the doctor and they just don't listen'-F8. Few participants understood the salience of the disease enough to strike some sort of balance between COPD and other comorbidities during their general practitioner consultations.

\section{v) Interplay of COPD and comorbidities}

Comorbidity count of the participants ranged from 1 to 7 , with most taking medication for all their health conditions. Of a total 32 comorbidities, as shown in Figure 2, hypercholesterolaemia was the most prevalent (31\%), followed by hypertension and arthritis (25\%), depression (12\%) and diabetes (9\%).

Among the 17 participants, only 29\% gave the highest priority to COPD among all their health conditions. The importance of COPD mostly outweighed hypercholesterolaemia, hypertension and depression in this study, but was overshadowed by diabetes. For those most impacted by arthritis, COPD was given higher priority. 'That (COPD) limits your whole life, doesn't it really... because you need the air'-F2.

Overall, the experience of multiple health conditions was challenging in terms of consuming various medications and scheduling different healthcare appointments. 'You try all these (treatments), they work for a little while but they don't solve the problem'-F7.

\section{DISCUSSION}

\section{Main findings}

Analysis of our study results was driven by a constructivist interpretation of the participants' experiences of a recent COPD diagnosis in the context of multimorbidity. Our findings indicated that though the participants accepted the diagnosis, they did not recognise the importance of COPD, mostly due to low awareness of the disease and its long-term implications. This may explain the participants' suboptimal self-management capacity of COPD and underutilisation of relevant healthcare. Low prioritisation of COPD in view of other comorbidities could be explained by the fact that the participants were recently diagnosed and also because most of them had mild severity of the disease. The relationship between COPD and its comorbidities was also an important aspect of our study and has implications for COPD management in primary care.

Strengths and limitations of this study

We believe this study is the first to explore patients' perspectives regarding the experience and impact of a new diagnosis of COPD in primary care patients with comorbidities. All the study participants had early-stage COPD, unlike other qualitative studies, which have focused on patients in its later stages. ${ }^{1922}$ The interviews served as a platform for some of the participants to 
voice their health-related concerns. The elapsed time between COPD diagnosis and this study, which spanned 5-24 months, might have affected the participants' recollections of their exact experience. However, this timespan probably resulted in obtaining richer perspectives about living with COPD. As most of the participants were of Caucasian origin, similar studies with diverse ethnic backgrounds are recommended.

Interpretation of findings in relation to previously published work Low knowledge has been reported even in later-stage COPD patients by Barr et al., ${ }^{20}$ which is a concern for healthcare providers. As many participants gave COPD lower priority than their comorbidities, there is a need for promotion of better awareness among patients. ${ }^{22,23}$ As the participants had either mild or moderate COPD, limitation of functional capacity and impairment of quality of life were relatively less than that reported in other studies where patients had higher severity of COPD. ${ }^{21,22}$

In line with previous research, gender disparity was evident among the participants in terms of reporting functional limitation. ${ }^{32,33}$ The fact that our female participants seemed more open towards seeking professional help and had better success with smoking cessation than their male counterparts, is on a par with the findings of Watson et al., ${ }^{32}$ who found that women are more likely to report dyspnoea and seek smoking cessation advice. On the other hand, work-related stress coupled with anxiety and/or depression, complicated smoking cessation for both genders in our study, which was also reported by DiNicola et al. $^{34}$

Misconception and confusion about the interrelated behaviour of COPD and asthma poses a concern for management of patients with an asthma-COPD overlap. ${ }^{35}$ Participants with discordant comorbidities, such as diabetes or cancer, were able to prioritise their conditions more clearly than those with concordant comorbidities like asthma or sleep apnoea. Such competing priorities add to the confusion and helplessness perceived from our interviews, which was also noted by Sheridan et al. ${ }^{36}$

In spite of limited understanding of COPD, all our participants understood cigarette smoking to be its main cause. This differs from the findings of Boeckxstaens et al., ${ }^{19}$ who reported a reluctance among their participants to accept the association between smoking and their COPD. Yet, some of our participants seemed very passive in seeking help for smoking cessation. This could be due to their sense of shame and self-blame ${ }^{24,36}$ for having contributed to the occurrence of COPD.

Socioeconomic status of the participants had an impact on their health perspectives. Level of education seemed to influence how participants experienced and managed their various health conditions. Those with tertiary education seemed to better understand the significance and implications of COPD and prioritise it more logically in view of their other comorbidities, as noted by Hudon et al. ${ }^{37}$ Participants living with family were better motivated and had a more positive outlook towards tackling their health conditions, with greater control over smoking and their overall health behaviour. ${ }^{19,38}$

A need for person-centredness, which has also been reported by McDonald et al., ${ }^{35}$ is vital for the management of COPD with comorbidities and can improve patient adherence. In spite of multimorbidity and increased health burden following COPD diagnosis, there was a positive vein among our participants. This resulted in better adaptation and coping to the new diagnosis than expected, a finding that has also been noted in previous studies. $^{19,39}$

Implications for future research and practice

Low awareness and prioritisation of COPD, suboptimal selfmanagement capacity and complications presented by conflicting comorbidities were the highlights of this study. Implications of these findings for healthcare providers in terms of managing COPD patients with comorbidities are a need for acceptance and incorporation of patient perspectives in primary care, and development of holistic care plans. In terms of future research, our study could be a base for interventions aimed at improving patient knowledge and self-management capacity. Also, the patient-provider relationship needs to be explored in future studies on COPD with multimorbidity.

\section{Conclusions}

Our qualitative study explored the perspectives of primary care patients regarding the impact of a new diagnosis of COPD in the context of multimorbidity. The key findings were limited understanding and low prioritisation of COPD in view of other comorbidities. These findings suggest the need for tailored education to increase awareness about COPD and activation of these patients to improve their self-management capacity in the context of multimorbidity. As COPD is typically diagnosed and managed in primary care, this is the most suitable setting for personalised management that incorporates patient's perspectives.

\section{ACKNOWLEDGEMENTS}

The authors thank the participants of this study for making it possible. Assistance by Dr Oshana Hermiz in verifying some of the baseline data is appreciated.

\section{CONTRIBUTIONS}

$\mathrm{HH}$ and NZ conceptualised and designed the research project. SA performed sampling, participant recruitment and data collection, coding, analysis and interpretation, drafted the manuscript and coordinated its revision. $\mathrm{HH}, \mathrm{SD}$ and NZ supervised data collection, participated in data analysis and interpretation, critically reviewed and revised the manuscript, and approved the final draft.

\section{COMPETING INTERESTS}

The authors declare no conflict of interest.

\section{FUNDING}

This study was covered by the National Health and Medical Research Council's funding for the PELICAN trial under Project Grant No. 630421.

\section{REFERENCES}

1 Osthoff M, Jenkins C, Leuppi JD. Chronic obstructive pulmonary disease-a treatable disease. Swiss Med Wkly 2013; 143: w13777.

2 Toelle BG, Xuan W, Bird TE, Abramson MJ, Atkinson DN, Burton DL et al. Respiratory symptoms and illness in older Australians: the Burden of Obstructive Lung Disease (BOLD) study. Med J Aust 2013; 198: 144-148.

3 Sekine $Y$, Katsura H, Koh E, Hiroshima K, Fujisawa T. Early detection of COPD is important for lung cancer surveillance. Eur Respir J 2012; 39: 1230-1240.

4 Vestbo J, Hurd SS, Agusti AG, Jones PW, Vogelmeier C, Anzueto A et al. Global Strategy for the diagnosis, management and prevention of chronic obstructive pulmonary disease: GOLD executive summary. Am J Respir Crit Care Med 2013; 187: $347-365$.

5 Akker MVD, Buntinx F, Knottnerus JA. Comorbidity or multimorbidity: what's in a name? A review of literature. Eur J Gen Pract 1996; 2: 65-70.

6 Luijks $\mathrm{H}$, Schermer $\mathrm{T}$, Bor $\mathrm{H}$, van Weel C, Lagro-Janssen T, Biermans $M$ et al. Prevalence and incidence density rates of chronic comorbidity in type 2 diabetes patients: an exploratory cohort study. BMC Med 2012; 10: 128

7 Kelly SO, Smith SM, Lane S, Teljeur C, Dowd TO. Chronic respiratory disease and multimorbidity: Prevalence and impact in a general practice setting. Respir Med 2011; 105: 236-242.

8 Mercer SW, Smith SM, Wyke S, Dowd TO, Watt GC. Multimorbidity in primary care: developing the research agenda. Fam Pract 2009; 26: 79-80.

9 Fortin M, Bravo G, Hudon C, Vanasse A, Lapointe L. Prevalence of multimorbidity among adults seen in family practice. Ann Fam Med 2005; 3: 223-228. 
10 Jowsey T, McRae IS, Valderas JM, Dugdale P, Phillips R, Bunton R et al. Time's up. descriptive epidemiology of multi-morbidity and time spent on health related activity by older australians: a time use survey. Plos One 2013; 8: e59379.

11 Brett T, Arnold-Reed DE, Popescu A, Soliman B, Bulsara MK, Fine H et al. Multimorbidity in patients attending 2 Australian primary care practices. Ann Fam Med 2013; 11: 535-542.

12 Sundh J, Stallberg B, Lisspers K, Montgomery SM, Janson C. Co-morbidity, body mass index and quality of life in COPD using the Clinical COPD Questionnaire. COPD 2011; 8: 173-181.

13 Burgel PR, Escamilla R, Perez T, Carré P, Caillaud D, Chanez P et al. Impact of comorbidities on COPD-specific health-related quality of life. Respir Med 2013, 107: 233-241.

14 Monninkhof E, Aa MVD, Valk PVD, van der Palen J, Zielhuis G, Koning $\mathrm{K}$ et al. A qualitative evaluation of a comprehensive self-management programme for COPD patients: effectiveness from the patients' perspective. Patient Educ Couns 2004; 55: 177-184.

15 Chatila WM, Thomashow BM, Minai OA, Criner GJ, Make BJ. Comorbidities in chronic obstructive pulmonary disease. Proc Am Thorac Soc 2008; 5: 549-555.

16 Corsonello A, Incalzi RA, Pistelli R, Pedone C, Bustacchini S, Lattanzio F. Comorbidities of chronic obstructive pulmonary disease. Curr Opin Pulm Med 2011; 17: S21-S28.

17 García-Olmos L, Alberquilla A, Ayala V, García-Sagredo P, Morales L, Carmona M et al. Comorbidity in patients with chronic obstructive pulmonary disease in family practice: a cross sectional study. BMC Fam Pract 2013; 14: 11.

18 Anecchino C, Rossi E, Fanizza C, De Rosa M, Tognoni G, Romero M. Prevalence of chronic obstructive pulmonary disease and pattern of comorbidities in a general population. Int J Chron Obstruct Pulmon Dis 2007; 2: 567-574.

19 Boeckxstaens P, Deregt M, Vandesype P, Willems S, Brusselle G, Sutter AD. Chronic obstructive pulmonary disease and comorbidities through the eyes of the patient Chron Respir Dis 2012; 9: 183-191.

20 Barr RG, Celli BR, Mannino DM, Petty T, Rennard SI, Sciurba FC et al. Comorbidities, patient knowledge, and disease management in a national sample of patients with COPD. Am J Med 2009; 122: 348-355.

21 Hernandez P, Balter M, Bourbeau J, Hodder R. Living with chronic obstructive pulmonary disease: a survey of patients' knowledge and attitudes. Respir Med 2009; 103: 1004-1012.

22 Barnett M. Chronic obstructive pulmonary disease: a phenomenological study of patients' experiences. J Clin Nurs 2005; 14: 805-812.

23 Sayiner A, Alzaabi A, Obeidat NM, Nejjari C, Beji M, Uzaslan E et al. Attitudes and beliefs about COPD: data from the BREATHE study. Respir Med 2012; 106 S60-S74.

24 Arne $M$, Emtner $M$, Janson S, Wilde-Larrson B. COPD patients' perspectives at the time of diagnosis: a qualitative study. Prim Care Respir J 2007; 16: 215-221.

25 Bunker JM, Reddel HK, Dennis SM, Middleton S, Van Schayck C, Crockett AJ et al. A pragmatic cluster randomized controlled trial of early intervention for chronic obstructive pulmonary disease by practice nurse-general practitioner teams: Study Protocol. Implement Sci 2012; 7: 83.

26 Ahern K. Informed consent: are researchers accurately representing risks and benefits? Scand J Caring Sci 2012; 26: 671-678.

27 Morse JM. The significance of saturation. Qual Health Res 1995; 5: 147.

28 Liamputtong, P. Qualitative Research Methods, 3rd edn. Oxford University Press: Oxford, MI, USA, 2009.

29 Appleton JV, King L. Journeying from the philosophical contemplation of constructivism to the methodological pragmatics of health services research. $J$ Adv Nurs 2002; 40: 641-648.

30 Closing the Gap, Australia, http://www.healthinfonet.ecu.edu.au/closing-the-gap.

31 Newstart Allowance, Australia, http://www.humanservices.gov.au/customer/ser vices/centrelink/newstart-allowance.

32 Watson L, Vestbo J, Postma DS, Decramer M, Rennard S, Kiri VA et al. Gender differences in the management and experience of chronic obstructive pulmonary disease. Respir Med 2004; 98: 1207-1213.

33 Naberan K, Azpeitia A, Cantoni J, Miravitlles M. Impairment of quality of life in women with chronic obstructive pulmonary disease. Respir Med 2012; 106: 367-373.

34 DiNicola G, Julian L, Gregorich SE, Blanc PD, Katz PP. The role of social support in anxiety for persons with COPD. J Psychosom Res 2013; 74: 110-115.

35 McDonald VM, Higgins I, Gibson PG. Insight into older peoples' healthcare experiences with managing COPD, asthma, and asthma-COPD overlap. J Asthma 2013; 50: 497-504.

36 Sheridan N, Kenealy T, Salmon E, Rea H, Raphael D, Schmidt-Busby J. Helplessness, self blame and faith may impact on self management in COPD: a qualitative study. Prim Care Respir J 2011; 20: 307-314.

37 Hudon $C$, Fortin M, Poitras ME, Almirall J. The relationship between literacy and multimorbidity in a primary care setting. BMC Fam Pract 2012; 13: 33.

$38 \mathrm{Holm} \mathrm{KE}$, Bowler RP, Make BJ, Wamboldt FS. Family relationship quality is associated with psychological distress, dyspnea, and quality of life in COPD. COPD 2009; 6: 359-368.

39 Kirby SE, Dennis SM, Bazeley P, Harris MF. Activating patients with chronic disease for self-management: comparison of self-managing patients with those managing by frequent readmissions to hospital. Aust J Prim Health 2013; 19: 198-206.

C) $($ ) This work is licensed under a Creative Commons Attributioncc) NonCommercial-NoDerivs 4.0 International License. The images or other third party material in this article are included in the article's Creative Commons license, unless indicated otherwise in the credit line; if the material is not included under the Creative Commons license, users will need to obtain permission from the license holder to reproduce the material. To view a copy of this license, visit http:// creativecommons.org/licenses/by-nc-nd/4.0/ 